\title{
HIPPARCOS MAIN MISSION PHOTOMETRIC PROCESSING
}

\author{
F. Mignard, M. Froeschlé, J.L. Falin \\ Observaloire de la Côte d'Azur / CERGA \\ Av. Copernic, F06130 GRASSE
}

\begin{abstract}
In addition to its astrometric capabilitie the Hipparcos main detector proves to be a good phototometer. The main features of the reduction are outlined altogether with a presentation of some conspicuous results which already provide good clues as to the quality of the final results when more than three years of data are analysed.
\end{abstract}

\section{INTRODUCTION}

The Hipparcos satellite launched by ESA on August 1989, has been primarily designed as a powerful instrument to carry out space astrometry at the milliarcsecond level. During the preparation of the reduction it has become apparent that the satellite had also powerful capabilities to measure accurately the amount of light received from the stars. It is the aim of the photometric processing to extract the photometric information from the data and to carry out further analysis to assess the variability or constancy of the star energy output.

\section{SIGNAL PROCESSING}

This photometry is essentially achieved by counting the number of photons crossing the entrance pupil per unit of time and collected by the detector located behind the main grid. Then these numbers are converted into a magnitude defined by a set of standard stars and a model of the instrument response. The latter depends essentially on the sensitivity change, from point to point on the grid, on the star color, and also on the epoch because of the overall sensitivity decreases as the mission progresses. This instrumental parameters are calibrated with great care about once every ten hours and are used afterwards to determine the Hipparcos magnitude of non-reference stars.

The global time signal, $S(t)$, is,

$$
S(t)=I+B+I M \cos (\omega t+\phi)+I N \cos (2 \omega t+\psi)
$$

where $I$ is the total intensity, $B$ the unmodulated background, $M$ and $N$ the modulation coefficients of the first and second harmonic respectively and $\phi$ and $\psi$ the corresponding phases. For a single

418

J. Bergeron (ed.), Highlights of Astronomy, Vol. 9, 418-421.

(C) 1992 IAU. Printed in the Netherlands. 
star we have typically $M=0.72$ and $N=0.25$. Thus the photometric information appears in $I$, $I M$ and $I N$ and must be extracted from these three parameters. Once this is done the Hipparcos magnitude is computed with the usual definition

$$
H_{p}=-2.5 \log _{10} \frac{I}{I_{0}}
$$

where $I_{0}$ is an arbitrary reference intensity.

Each star crossing of the Hipparcos grid results in one determination of the magnitude; for a given star, observations are repeated at different interval $(20 \mathrm{~m}, 2 \mathrm{~h}, 6$ weeks) over the mission duration, the peculiarities of which are tied to the Hipparcos unusual scanning law. Altogether an average star will be observed about 150 times during the Hipparcos mission. The precision obtained per transit are shown in table 1. as a function of the star magnitude.

Table 1. Photometric precision for one field transit

\begin{tabular}{lcccccccccc}
\hline Magnitude & 4 & 5 & 6 & 7 & 8 & 9 & 10 & 11 & 12 & 13 \\
Precision (0.001 mag) & 2 & 4 & 4 & 7 & 10 & 14 & 17 & 30 & 40 & 70 \\
\hline
\end{tabular}

\section{ANALYSIS OF THE RESULTS}

From this basic data several analysis are performed. If the measured intensities show no statistically significant variation with the time, one computes a single value of the star magnitude from a weighted mean of the intensities determined at every transit. On the other hand when the signal displays regular or irregular variations, detected by appopriated statistical tests, the results of individual crossing are output for further investigations.
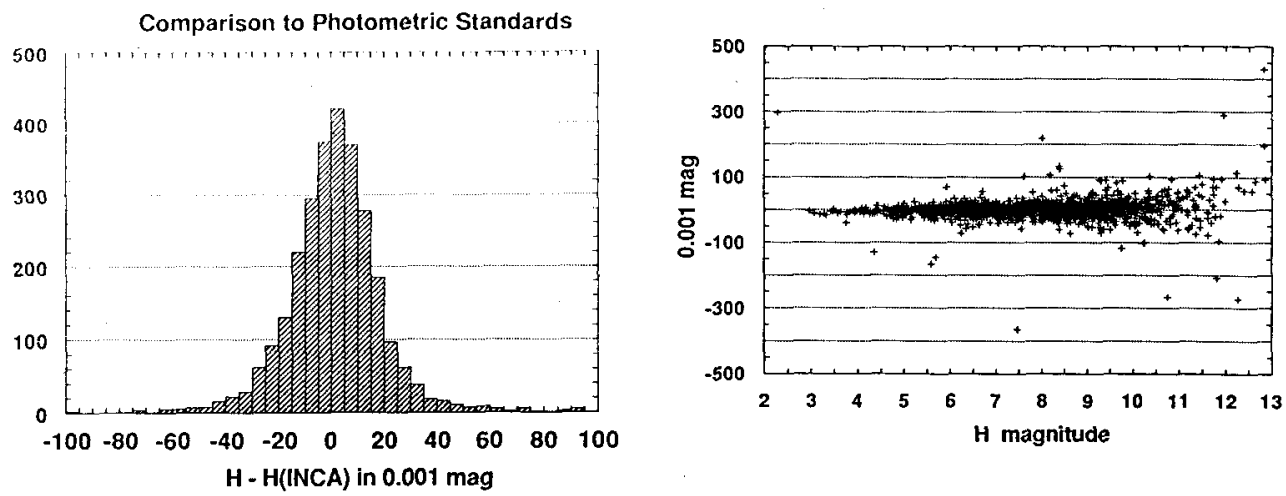

Fig. 1 1.a Distribution of the errors in magnitude determination 1.b Same data as a function of the magnitude.

The main results based on the first six months of observations are summarized in fig. 1-3. First we analyse in figure 1 the error in the magnitude of the photometric standard stars by comparing the 
Hipparcos determination to the reference values available in the Hipparcos Input Catalogue. Altogether a set of 14000 stars have been carefully measured in several color before the mission and their a priori magnitude in the Hipparcos band is known with a standard deviation of 15 millimagnitude. The histogram in fig.1a shows that the reduction is properly adjusted to the Hipparcos band defined by the set of standard stars. The dispersion in the diagram is essentially due to the scaterring in the materialization of the Hipparcos magnitude scale by the set of photometric standard stars. The same data is plotted as a function of the magnitude in fig. $1 \mathrm{~b}$.

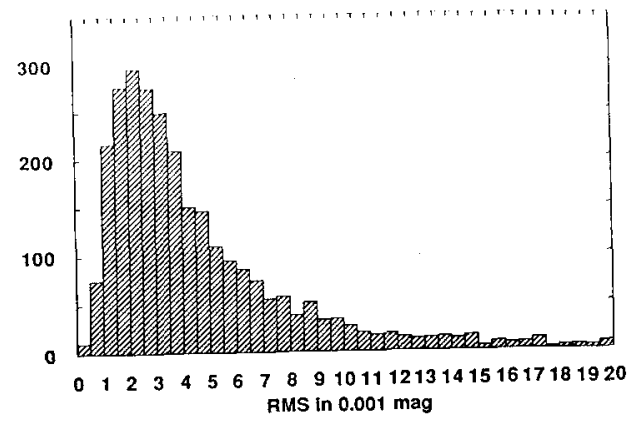

Figure 2. Precisions achieved in the photometric observations.

The diagram in fig. 2 gives the actual distribution of the mean error for stars which have been observed over at least 5 transits. The mean error, of the order of few millimagnitudes, is estimated from the observed scattering of the individual results, and therefore is in fact a measure of the consistency of the reduction with the time.

The plot in figure 3.a illustrates the time stability of the solution in showing about 50 individual results obtained over 4 days on a constant star. Despite the results are based on 8 independant calibration of the instrument sensitivity over this period, the processing sticks to a well defined Hipparcos scale.
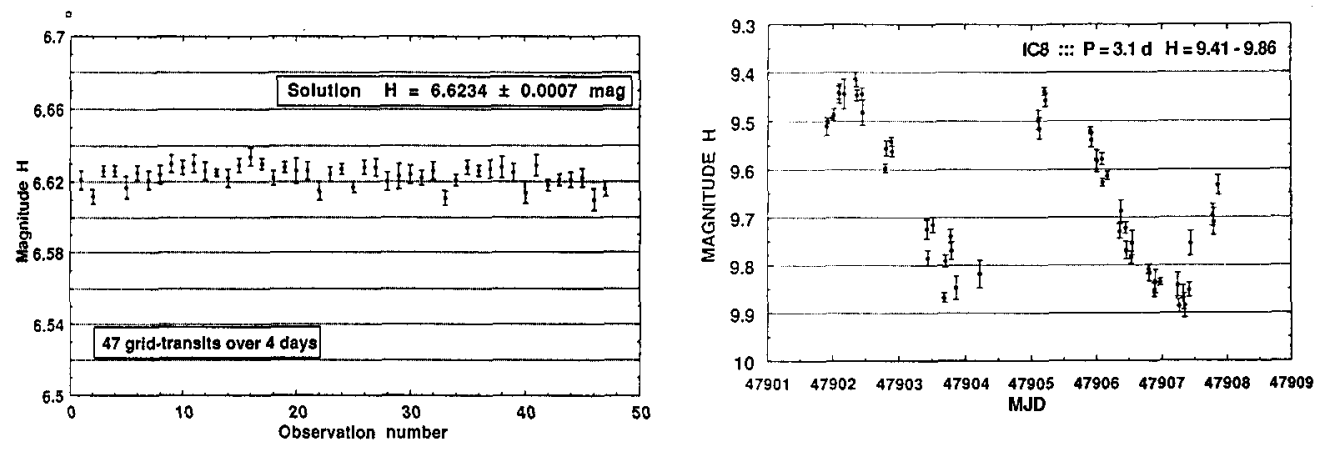

Fig. 3 3.a Time stability of the photometric solution 3.b Observation of a Cepheid by Hipparcos

Conversely we show in figure 3.b the observation of a known cepheid over nearly two cycles. It is hoped that many such stars will be observed during the mission and that less conspicuous variables will be detected by the satellite. 